\title{
Búsqueda de Catalizadores Sólidos Básicos para la Producción de Biodiesel
}

\author{
Mónica Becerra, Aristóbulo Centeno, Sonia A. Giraldo \\ Universidad Industrial de Santander (UIS), Centro de Investigaciones en Catálisis (CICAT), \\ Escuela de Ingeniería Química, Carrera 27 Calle 9, Bucaramanga-Colombia \\ (e-mail: sgiraldo@uis.edu.co)
}

Recibido Sept. 11, 2009; Aceptado Nov. 24, 2009; Versión Final recibida Dic. 10, 2009

\section{Resumen}

Esta investigación tuvo como objetivo la búsqueda de catalizadores sólidos básicos activos y estables que contribuyan al desarrollo de sistemas de reacción heterogéneos para producción limpia de biodiesel. Se prepararon y evaluaron en la transesterificación de triacetina tres tipos de catalizadores: $\mathrm{CaO}, \gamma-\mathrm{Al}_{2} \mathrm{O}_{3}$ modificada con $\mathrm{Na}$ y $\mathrm{K}$, y $\mathrm{MgO}$ modificado con $\mathrm{Na}, \mathrm{Li}, \mathrm{Rb}$ y $\mathrm{Cs}$. Se analizó la influencia de las propiedades texturales, tipo y cantidad de metal impregnado y la activación con $\mathrm{N}_{2}$, sobre la basicidad y el comportamiento catalítico. Se estudió la estabilidad de estos materiales mediante absorción atómica y reusos consecutivos. Los resultados mostraron que la impregnación de metales no conduce a la formación de sólidos estables, ya que éstos se lixivian en el medio de reacción. El CaO mantuvo una elevada actividad catalítica durante cinco ciclos de reacción convirtiéndolo en un material con alto potencial para ser utilizado en un proceso industrial.

Palabras claves: transesterificación, triacetina, catalizadores sólidos básicos, biodiesel

\section{Searching for Solid base Catalysts for Biodiesel Production}

\begin{abstract}
This work aimed to find active and stable solid base catalysts for clean biodiesel production using heterogeneous reaction systems. Three types of catalysts were synthesized and evaluated in the transesterification of triacetin: $\mathrm{CaO}, \gamma-\mathrm{Al}_{2} \mathrm{O}_{3}$ modified with $\mathrm{Na}$ and $\mathrm{K}$, and $\mathrm{MgO}$ modified with $\mathrm{Na}, \mathrm{Li}$, $\mathrm{Rb}$ and $\mathrm{Cs}$. The influence of the textural proprieties, the type and amount of alkali metal impregnated and the activation with $\mathrm{N}_{2}$ on the basicity and the catalytic behavior were analyzed. Stability of catalysts was analyzed using atomic absorption measurements and reuse reactions. Results showed that metal impregnation does not lead to the formation of stable solids, because they present lixiviation in the reaction system. The $\mathrm{CaO}$ maintained a high catalytic activity during five reaction cycles showing its high potential of its use in an industrial process.
\end{abstract}

Keywords: trransesterification, triacetin, solid base catalysts, biodiesel 


\section{INTRODUCCIÓN}

En la actualidad los procesos industriales para la producción de biodiésel emplean sistemas de reacción en fase homogénea. Estos procesos son relativamente rápidos y presentan altas conversiones, sin embargo, el catalizador no puede ser reutilizado y se requieren etapas de neutralización y purificación que aumentan los costos de producción y generan importantes efluentes contaminantes. La catálisis heterogénea se presenta como una alternativa promisoria para la solución integral a los problemas operacionales y ambientales que presenta la producción convencional de biodiésel. A escala de laboratorio, una variedad de sólidos básicos que incluye zeolitas modificadas, hidrotalcitas, metales alcalinos soportados y óxidos alcalinotérreos, han sido probados en la transesterificación con el fin de solventar los problemas mencionados y simplificar el proceso de producción (Ebiura et al., 2005; Xi et al., 2008; Arzamendi et al., 2008).

Algunos autores han demostrado que la impregnación de metales en soportes como la $\gamma-\mathrm{Al}_{2} \mathrm{O}_{3}$ (Arzamendi et al., 2007; Xie et al., 2006) y el MgO (Kim et al., 2004) conduce a la formación de materiales con alta actividad catalítica en la transesterificación. Si bien estos catalizadores parecen ser candidatos idóneos para la sustitución de un proceso catalítico homogéneo por uno heterogéneo, su estabilidad no ha sido estudiada en detalle. Es importante prestar una mayor atención a la estabilidad de los sistemas catalíticos sólidos en las reacciones de transesterificación, ya que si las especies activas se desprenden en el medio de reacción actuarán como un sistema homogéneo y el catalizador sólido presentará un menor número de sitios activos disponibles para reacciones sucesivas, haciendo imposible la reactivación y anulando las posibles ventajas de usar estos sólidos como catalizadores para este proceso.

Esta investigación está dirigida a la búsqueda de catalizadores sólidos activos y estables que se puedan utilizar en el desarrollo de procesos de producción de ésteres. Se estudió la efectividad de catalizadores sólidos básicos sintetizados a partir $\gamma-\mathrm{Al}_{2} \mathrm{O}_{3}$ modificada con $\mathrm{Na}$ y $\mathrm{K}$, MgO modificado con $\mathrm{Na}, \mathrm{Li}, \mathrm{Rb}$ y $\mathrm{Cs}$, y $\mathrm{CaO}$, en la producción de acetato de etilo a partir de triacetina. Se hizo énfasis en la búsqueda de una actividad aceptable comparada con la de los sistemas homogéneos y sobre la estabilidad de los materiales preparados. La estabilidad se analizó en reacciones de reuso o determinando el contenido de metal después de extracciones Soxhlet con etanol. Además, se examinó la influencia de las características texturales y de la basicidad de los catalizadores sobre la actividad en la transesterificación de la triacetina con el fin de establecer una correlación. La triacetina fue seleccionada como molécula modelo porque sólo difiere de los triglicéridos comunes en el tamaño, por su disponibilidad en forma pura y porque su simplicidad química y estructural facilita la identificación y cuantificación de los productos de reacción, manteniendo la misma funcionalidad compartida por todos los triglicéridos

\section{MATERIALES Y MÉTODOS}

Todos los catalizadores se prepararon y evaluaron utilizando reactivos analíticos. La triacetina (99\%), el dodecano (>99\%), el $\mathrm{Cs}_{2} \mathrm{CO}_{3}(99 \%)$ y el $\mathrm{Rb}_{2} \mathrm{CO}_{3}(99 \%)$ de Sigma-Aldrich. El etanol (>99.5\%), el $\mathrm{CaO}(\geq 97,0 \%)$, el $\mathrm{MgO}(99 \%)$, el $\mathrm{NaOH}(99 \%)$, el $\mathrm{KOH}(99 \%)$, el KF $(99 \%)$ y el $\mathrm{LiOH}(99 \%)$ de Merck. El $\mathrm{Na}_{2} \mathrm{CO}_{3}(99 \%)$ de Carlo Erba, y la $\mathrm{y}-\mathrm{Al}_{2} \mathrm{O}_{3}$ de Procatalyse. El CaO en trozos pequeños ( 3$20 \mathrm{~mm}$ ), fue pulverizado y empleado como catalizador sin ningún tratamiento adicional.

\section{Sintesis de los catalizadores}

Se preparó una serie de catalizadores con base en $\gamma-\mathrm{Al}_{2} \mathrm{O}_{3}$ y una segunda en $\mathrm{MgO}$. Estos materiales se modificaron con tratamientos hidrotérmicos y con metales alcalinos, variando la sal precursora, el contenido de metal y la temperatura de calcinación, con el fin de mejorar sus características texturales y sus propiedades básicas, y obtener catalizadores estables. Para la primera serie, la $\gamma$ $\mathrm{Al}_{2} \mathrm{O}_{3}$ fue triturada, pulverizada e impregnada con soluciones acuosas de $\mathrm{NaOH}, \mathrm{Na}_{2} \mathrm{CO}_{3}, \mathrm{KOH}$ o $\mathrm{KF}$ de diferente concentración para obtener contenidos de metal de 1, 6, 8 y 11\% en el catalizador. $6 \mathrm{~g}$ de cada sólido obtenido se secaron en flujo de aire $(50 \mathrm{ml} / \mathrm{min})$ a $120^{\circ} \mathrm{C}$ por $12 \mathrm{~h}$ y se calcinaron a $350^{\circ} \mathrm{C} \circ 500^{\circ} \mathrm{C}$ por $2 \mathrm{~h}$ aumentando la temperatura a razón de $2^{\circ} \mathrm{C} / \mathrm{min}$. Los catalizadores de esta serie se designan con la sigla $\mathrm{M}(\mathrm{x}) / \gamma-\mathrm{Al}_{2} \mathrm{O}_{3}$, donde $\mathrm{M}$ indica la sal precursora, $\mathrm{x}$ la cantidad nominal 
(\% masa) de metal impregnado. Para los catalizadores de la segunda serie, el MgO comercial $\left(\mathrm{MgO}_{\mathrm{C}}\right)$ fue sometido previamente a un tratamiento hidrotérmico de acuerdo al protocolo utilizado por Di Cosimo et al. (1996), con el objetivo de aumentar el área superficial. A $10 \mathrm{~g}$ de $\mathrm{MgO}_{\mathrm{C}}$ se adicionaron lentamente $100 \mathrm{ml}$ de agua destilada bajo agitación constante a temperatura ambiente. La mezcla se llevó a $80^{\circ} \mathrm{C}$ y se mantuvo a esta temperatura por $4 \mathrm{~h}$. El sólido se filtró y se secó a $110^{\circ} \mathrm{C}$ por $12 \mathrm{~h}$. La muestra resultante fue tratada térmicamente a $350^{\circ} \mathrm{C}$ por $2 \mathrm{~h}$ y calcinada a $500^{\circ} \mathrm{C}$ por $8 \mathrm{~h}$ con flujo de aire de $30 \mathrm{ml} / \mathrm{min}$. El sólido obtenido se denomina MgO. Muestras de $\mathrm{MgO}$ se modificaron con $\mathrm{Na}$, $\mathrm{Li}, \mathrm{Cs}$ o $\mathrm{Rb}$, mediante impregnación húmeda incipiente con soluciones acuosas de $\mathrm{NaOH}, \mathrm{LiOH}, \mathrm{Cs}_{2} \mathrm{CO}_{3}$ o de $\mathrm{Rb}_{2} \mathrm{CO}_{3}$ a la concentración correspondiente para obtener un $0,5 \%$ de metal en el catalizador. $6 \mathrm{~g}$ de las muestras que contenían $\mathrm{Na}$ o Li se secaron durante $12 \mathrm{~h}$ a $110^{\circ} \mathrm{C}$ y se calcinaron a $350^{\circ} \mathrm{C}$ por $2 \mathrm{~h}$ en flujo de aire $(30 \mathrm{ml} / \mathrm{min})$ y posteriormente a $500^{\circ} \mathrm{C}$ por $8 \mathrm{~h}$ aumentando la temperatura a razón de $2^{\circ} \mathrm{C} / \mathrm{min}$. Las muestras con Cs o Rb se calcinaron en mufla a $900^{\circ} \mathrm{C}$ por $4 \mathrm{~h}$, debido a que sus sales precursoras se descomponen por encima de $800^{\circ} \mathrm{C}$. Los catalizadores de esta segunda serie se denominaron $\mathrm{M} / \mathrm{MgO}-\mathrm{T}_{\mathrm{c}}$, donde $\mathrm{M}$ es el metal y $\mathrm{T}_{\mathrm{c}}$ la temperatura de calcinación.

\section{Caracterización de los catalizadores}

Las propiedades texturales de los catalizadores, área específica BET $\left(\mathrm{A}_{\mathrm{BET}}\right)$, volumen total de poros $\left(V_{\mathrm{P}}\right)$ y tamaño de poros $\left(\mathrm{D}_{\mathrm{P}}\right)$, se midieron con base en las isotermas de adsorción-desorción de $\mathrm{N}_{2}$ obtenidas en un equipo NOVA 1200 (Quantachrome). Las muestras fueron previamente desgasificadas en vacío a $100^{\circ} \mathrm{C}$ durante $12 \mathrm{~h}$.

La fortaleza básica $\left(H_{-}\right)$y la basicidad de los catalizadores se determinaron por el método de titulación de Hammett. Se mezcló $0,1 \mathrm{~g}$ de catalizador con $2 \mathrm{ml}$ de una solución del indicador. Esta suspensión fue valorada con ácido benzoico 0,01 M. Se emplearon los siguientes indicadores: azul de bromotimol $\left(H_{-}=7,2\right)$, fenolftaleína $\left(H_{-}=9,3\right), 2,4$-dinitroanilina $\left(H_{-}=15,0\right)$, 4-nitroanilina $\left(H_{-}=\right.$ 18,4) y 4-cloroanilina $\left(H_{-}=26,5\right)$.

\section{Evaluación de los catalizadores}

Se evaluó el comportamiento de todos los catalizadores en la transesterificación de triacetina con etanol en un sistema de reacción tipo batch, compuesto por un balón de tres bocas con sistemas de condensación, muestreo, control de temperatura y agitación magnética. La carga del reactor $(60 \mathrm{ml})$ estaba compuesta por: etanol y triacetina en una relación molar igual a 10, dodecano utilizado como estándar interno para el análisis cromatográfico, y $3 \%$ en masa de catalizador. La temperatura de reacción fue de $60^{\circ} \mathrm{C}$. Se tomaron muestras de la mezcla de reacción $(\sim 0,2 \mathrm{ml})$ a diferentes tiempos. El volumen de reacción fue muy poco afectado por el muestreo, puesto que el volumen total de todas las muestras representó menos del $5 \%$ del volumen inicial de reacción. Las concentraciones de las muestras de reacción fueron determinadas por cromatografía de gases, utilizando un cromatógrafo HP 6890 equipado con detector FID, inyector split/splitless y una columna capilar HP-INNOWax $30 \mathrm{~m}$ x 0,32 $\mathrm{mm}$ x 0,25 $\mu \mathrm{m}$. La temperatura en el inyector y en el detector fue 255 y $260^{\circ} \mathrm{C}$, respectivamente. La programación del horno fue la siguiente: $70^{\circ} \mathrm{C}$ durante 4 min, luego una rampa de $20^{\circ} \mathrm{C} / \mathrm{min}$ hasta $100^{\circ} \mathrm{C}$ durante $1 \mathrm{~min}$ y finalmente una rampa de $50^{\circ} \mathrm{C} / \mathrm{min}$ hasta $240^{\circ} \mathrm{C}$ por $4 \mathrm{~min}$.

De la misma manera, se evaluó el comportamiento de catalizadores de la segunda serie sometidos previamente a un tratamiento de "activación", que consiste en remover con un gas inerte las moléculas adsorbidas de agua y $\mathrm{CO}_{2}$ de la superficie, las cuales neutralizan los sitios básicos (Bailly et al., 2005). La muestra de catalizador $(3 \mathrm{~g})$ se mantuvo a $500^{\circ} \mathrm{C}$ durante $12 \mathrm{~h}$ en flujo de $\mathrm{N}_{2}(30$ $\mathrm{ml} / \mathrm{min}$ ), se dejó enfriar bajo flujo de $\mathrm{N}_{2}$ y se transfirió al sistema de reacción evitando el menor contacto con el ambiente. Los catalizadores sometidos a este tratamiento se identifican con la letra $\mathrm{A}$ al final de su sigla.

La actividad de los catalizadores se expresa mediante la conversión de triacetina. La selectividad $\left(S_{i}\right)$ a diacetina, monoacetina o a glicerina se calculó utilizando la Ecuación 1. Se consideró el esquema de reacción normalmente aceptado, el cual presenta tres reacciones consecutivas: un triglicérido se combina con un alcohol para producir un alquil ester y glicerina, pasando por los intermedios di y monoglicérido (López et al., 2005). 
$S_{i}(\%)=\frac{C_{i}}{\sum{\stackrel{3}{C} C_{i}}_{i=1}} \times 100$

Donde $C_{i}$ es la concentración másica de cada componente.

La estabilidad de los catalizadores de la primera serie se evaluó determinando la efectividad de las impregnaciones de los metales. Se determinó el contenido de metal ( $\mathrm{Na}$ y $\mathrm{K}$ ) por absorción atómica (A.A) luego de realizarles una extracción Soxhlet con etanol durante $10 \mathrm{~h}$. Para el $\mathrm{CaO}$ y los catalizadores de la segunda serie, se estudió la estabilidad mediante reacciones de reuso. Para estas reacciones los catalizadores separados por filtración del medio de reacción fueron sometidos a lavados por extracción Soxhlet con etanol y luego secados a $60^{\circ} \mathrm{C}$ en estufa durante $12 \mathrm{~h}$. En las reacciones de reuso se empleó una cantidad de reactivos proporcional a la masa del catalizador recuperado, para mantener las condiciones de reacción iniciales.

\section{RESULTADOS Y DISCUSIÓN}

Determinación de la efectividad de la impregnación del metal en los catalizadores de la primera serie

En la Tabla 1 se presenta el contenido de metal medido por A.A para los catalizadores de la primera serie impregnados con 1 y $6 \%$ de metal, después de una extracción Soxhlet de $10 \mathrm{~h}$.

Tabla 1: Cantidad de metal en catalizadores de la primera serie después de extracción Soxhlet.

\begin{tabular}{|c|c|c|}
\hline \multirow[t]{2}{*}{ Catalizador } & \multicolumn{2}{|c|}{$\begin{array}{c}\% \text { en masa de metal a temperatura } \\
\text { de calcinación }\end{array}$} \\
\hline & $350^{\circ} \mathrm{C}$ & $500^{\circ} \mathrm{C}$ \\
\hline $\mathrm{NaOH}(6) / \gamma-\mathrm{Al}_{2} \mathrm{O}_{3}$ & 0,63 & 0,44 \\
\hline $\mathrm{Na}_{2} \mathrm{CO}_{3}(6) / \gamma-\mathrm{Al}_{2} \mathrm{O}_{3}$ & 0,93 & 0,59 \\
\hline $\mathrm{KOH}(6) / \gamma-\mathrm{Al}_{2} \mathrm{O}_{3}$ & 0,71 & 0,31 \\
\hline $\mathrm{KF}(6) / \gamma-\mathrm{Al}_{2} \mathrm{O}_{3}$ & 0,22 & 0,18 \\
\hline $\mathrm{NaOH}(1) / \gamma-\mathrm{Al}_{2} \mathrm{O}_{3}$ & 0,60 & 0,33 \\
\hline $\mathrm{Na}_{2} \mathrm{CO}_{3}(1) / \gamma-\mathrm{Al}_{2} \mathrm{O}_{3}$ & 0,95 & 0,34 \\
\hline $\mathrm{KOH}(1) / \gamma-\mathrm{Al}_{2} \mathrm{O}_{3}$ & 0,74 & 0,33 \\
\hline $\mathrm{KF}(1) / \gamma-\mathrm{Al}_{2} \mathrm{O}_{3}$ & 0,06 & 0,03 \\
\hline
\end{tabular}

En la Tabla 1 se observa que los catalizadores que retuvieron mayor cantidad de metal son $\mathrm{KOH}(1) / \gamma-\mathrm{Al}_{2} \mathrm{O}_{3}$ y $\mathrm{Na}_{2} \mathrm{CO}_{3}(1) / \gamma-\mathrm{Al}_{2} \mathrm{O}_{3}$ calcinados a $350^{\circ} \mathrm{C}$, con contenido de $\mathrm{K}$ y $\mathrm{Na}$ de 0,74 y $0,95 \%$ respectivamente. Los catalizadores con $6 \%$ y con contenidos más altos de metal antes de la extracción, cuyos resultados no se muestran en la Tabla 1, presentaron la mayor pérdida, reteniendo aproximadamente la misma cantidad que los impregnados con $1 \%$ de metal. La impregnación de la $\gamma$ $\mathrm{Al}_{2} \mathrm{O}_{3}$ con $\mathrm{KF}$ condujo a la formación del sólido más inestable de la primera serie.

\section{Características texturales de los catalizadores}

En la Tabla 2 se muestra las propiedades texturales de los catalizadores. Se observa que para los catalizadores de la primera serie que presentaron estabilidad, el área superficial presenta una leve disminución al adicionarle el metal comparada con la de la $\gamma-\mathrm{Al}_{2} \mathrm{O}_{3}$, de forma similar a lo reportado en la literatura (Kim et al., 2004). Por otro lado, para los catalizadores de la segunda serie se observa que el área del $\mathrm{MgO}$ es 4 veces mayor que la del $\mathrm{MgO}_{\mathrm{c}}$. Di Cosimo et al. (1996), entre otros, también reportan que el tratamiento hidrotérmico del $\mathrm{MgO}$ a alta temperatura aumenta su área superficial específica. Autores como Hee Lee et al. (2003), proponen que el incremento de la superficie del catalizador se presenta debido al aumento en la porosidad cuando el $\mathrm{MgO}$ es hidratado para formar $\mathrm{Mg}(\mathrm{OH})_{2}$, que al ser calcinado libera agua e impurezas presentes en la superficie, obteniéndose un $\mathrm{MgO}$ de mayor área superficial específica. 
Tabla 2: Propiedades texturales de los catalizadores.

\begin{tabular}{|l|l|c|c|}
\hline \multicolumn{1}{|c|}{ Catalizador } & $\begin{array}{c}\mathrm{A}_{\mathrm{BET}} \\
\left(\mathrm{m}^{2} / \mathrm{g}\right)\end{array}$ & $\begin{array}{c}\mathrm{V}_{\mathrm{p}} \\
\left(\mathrm{cm}^{3} / \mathrm{g}\right)\end{array}$ & $\begin{array}{c}\mathrm{D}_{\mathrm{p}} \\
(\AA)\end{array}$ \\
\hline $\mathrm{CaO}$ & 7 & 0,03 & 156 \\
\hline$\gamma-\mathrm{Al}_{2} \mathrm{O}_{3}$ & 208 & 0,60 & 116 \\
\hline $\mathrm{Na}_{2} \mathrm{CO}_{3}(1) / \gamma-\mathrm{Al}_{2} \mathrm{O}_{3}$ & 192 & 0,50 & 119 \\
\hline $\mathrm{KOH}(1) / \gamma-\mathrm{Al}_{2} \mathrm{O}_{3}$ & 198 & 0,53 & 117 \\
\hline $\mathrm{MgO}$ & 28 & 0,14 & 202 \\
\hline $\mathrm{MgO}$ & 123 & 0,50 & 159 \\
\hline $\mathrm{Na} / \mathrm{MgO}-500$ & 120 & 0,46 & 424 \\
\hline $\mathrm{Li} / \mathrm{MgO}-500$ & 8 & 0,04 & 420 \\
\hline $\mathrm{Cs} / \mathrm{MgO}-900$ & 42 & 0,26 & 434 \\
\hline $\mathrm{Rb} / \mathrm{MgO}-900$ & 43 & 0,23 & 424 \\
\hline $\mathrm{Li} / \mathrm{MgO}-950$ & 12 & 0,02 & 487 \\
\hline
\end{tabular}

Para los catalizadores de la segunda serie, $\mathrm{MgO}$ modificado con metales se observa que el proceso de impregnación y calcinación condujo a una disminución considerable del $A_{B E T}$, con respecto al $\mathrm{MgO}$. Este resultado está de acuerdo con lo reportado en la literatura, donde comprueban que al someter catalizadores preparados de forma análoga a temperaturas superiores a $600^{\circ} \mathrm{C}$, el $A_{B E T}$ disminuye considerablemente. Catalizadores $\mathrm{Li} / \mathrm{MgO}$ preparados convencionalmente, por impregnación de $\mathrm{MgO}$ con soluciones acuosas de sales de Li seguido de secado y calcinación, en general se caracterizan por bajas áreas superficiales $\left(<10 \mathrm{~m}^{2} / \mathrm{g}\right)$ (Leveles et al., 2003). Esta reducción drástica es causada principalmente por efectos de sinterización del sólido que genera bloqueo de los poros durante los procedimientos de preparación (Díez et al., 2000). Sólo mediante tratamiento a altas temperaturas se logra la incorporación de estos metales, haciendo posible que los iones metálicos se difundan en la red del $\mathrm{MgO}$, reemplacen los iones $\mathrm{Mg}^{2+}$ y de esta manera se generen sitios activos. La desventaja de este procedimiento es que la movilidad de las especies metálicas y las altas temperaturas facilitan la sinterización del MgO y la consecuente pérdida de área superficial (Trionfetti et al., 2006).

\section{Basicidad de los catalizadores}

La basicidad de la superficie de un óxido se relaciona generalmente con las propiedades de electrodonación de los aniones de oxígeno combinado, de manera que cuanto mayor es la carga parcial negativa en estos aniones, más básico será el óxido (Díez et al., 2000). La distribución de los sitios básicos de los catalizadores estudiados se muestra en la Fig. 1.

Para el $\mathrm{CaO}$, los indicadores azul de bromotimol y fenolftaleína mostraron su color básico rápidamente sobre el catalizador, pero a partir del siguiente indicador, 2,4-dinitroanilina $(H=15,0)$, no se presentó cambio de color a sus bases conjugadas. Por tanto, la fortaleza de los sitios básicos del $\mathrm{CaO}$ está en los rangos $7,2<H_{-}<9,3$ y 9,3<H_<15,0, destacándose el elevado número de sitios básicos presentes en este último intervalo. Los catalizadores estables de la primera serie sólo presentaron sitios básicos de fortaleza $7,2<H_{-}<9,3$ probablemente porque el contenido de metal es relativamente bajo. El tratamiento hidrotérmico aplicado al $\mathrm{MgO}_{\mathrm{c}}$ incrementó 4 veces la cantidad de sitios de fortaleza $7,2<H_{-}<9,3$, pero no generó sitios de mayor fuerza básica.

En la Fig. 1 también se observa el efecto en la basicidad del $\mathrm{MgO}$ al modificarlo con metales alcalinos. Se observa, en todos los casos, que al modificar el MgO aparecen sitios de fortaleza básica mayor. El Na/MgO-500 presenta la más amplia distribución de sitios básicos y además posee el mayor número de sitios en los rangos $7,2<H_{-}<9,3$ y $15,0<H_{-}<18,4$. Se observa que la naturaleza de los sitios básicos presente en los catalizadores modificados con metales varía con respecto a la temperatura de calcinación. Estos cambios en el número y naturaleza de los sitios se pueden entender según lo explica Hattori (2004), por el reordenamiento de la mayoría de los átomos de la superficie del sólido durante la calcinación. El incremento en la temperatura de calcinación genera sitios de tipo $\mathrm{O}^{2-}$ de diferente número de coordinación $\left(\mathrm{O}^{2-}{ }_{(5 \mathrm{c})}, \mathrm{O}^{2-}{ }_{(4 \mathrm{c})}\right.$ y $\left.\mathrm{O}^{2-}{ }_{(3 \mathrm{c})}\right)$ los cuales son responsables de las diferentes propiedades básicas. Temperaturas de calcinación de $500^{\circ} \mathrm{C}$ 
favorecen la capacidad de los sitios de iones de tipo $\mathrm{O}^{2-}$ de la superficie del $\mathrm{MgO}$, de donar su par de electrones para formar el respectivo óxido de metal, el cual otorga al sólido mejores características básicas; sin embargo, mayores incrementos en la temperatura de calcinación, hacen que estos sitios donde se forma el óxido, empiecen a ser inestables, tiendan a reorganizarse y desaparecer. Seguramente esto está ocasionando que el número de sitios básicos en los catalizadores calcinados a temperaturas superiores a $500^{\circ} \mathrm{C}$ sea muy bajo.

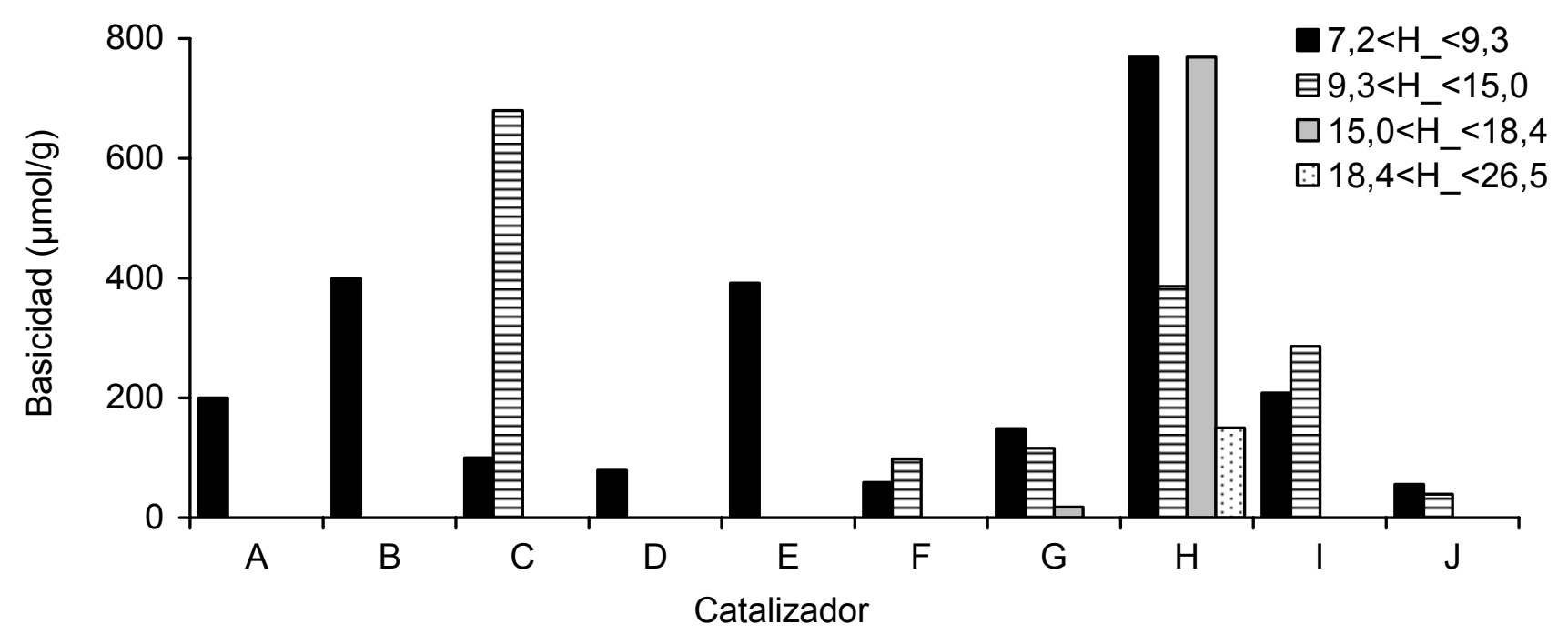

Fig. 1: Distribución de la fortaleza básica de los catalizadores; $(A) \mathrm{Na}_{2} \mathrm{CO}_{3}(1) / \gamma-\mathrm{Al}_{2} \mathrm{O}_{3}$, (B) $\mathrm{KOH}(1) / \gamma-$ $\mathrm{Al}_{2} \mathrm{O}_{3}$, (C) $\mathrm{CaO},(\mathrm{D}) \mathrm{MgO}_{\mathrm{C}}$, (E) MgO, (F) Cs/MgO-900, (G) Rb/MgO-900, (H) Na/MgO-500, (I) Li/MgO500 y (J) Li/MgO-950.

\section{Comportamiento de los catalizadores}

En la transesterificación usando catalizadores sólidos básicos, el primer paso de la reacción es la abstracción del protón del alcohol por los sitios básicos para formar el anión alcóxido. Este anión ataca un carbono carbonilo de la molécula del triglicérido, lo cual conduce a la formación de un intermedio tetraédrico (alcoxicarbonilo). Luego, este intermedio se divide en dos moléculas: alquil éster del ácido graso y el correspondiente anión del diglicérido (Kouzu et al., 2008). Los diglicéridos y monoglicéridos son convertidos por el mismo mecanismo que los triglicéridos, a una mezcla de alquil esteres y glicerina.

Se realizó una prueba catalítica inicial con la $\gamma-\mathrm{Al}_{2} \mathrm{O}_{3}$ y se comprobó que no hay reacción. En la Fig. 2 se presenta la conversión alcanzada en $4 \mathrm{~h}$ de reacción a $60^{\circ} \mathrm{C}$ con los catalizadores estudiados. Para los catalizadores de la primera serie se presentan los resultados de los que retuvieron mayor cantidad de metal después de ser sometidos a $10 \mathrm{~h}$ de extracción Soxhlet (ver Tabla 1). Se observa en la Fig. 2 que con el CaO se alcanza conversión completa de triacetina en el tiempo de reacción. Lo anterior tiene relación con el elevado número de sitios básicos de fortaleza $9,3<H \_<15,0$ que tiene el $\mathrm{CaO}$.

Para el caso del MgO se observa que el tratamiento hidrotérmico, aunque aumentó el $A_{B E T}$ y el número de sitios básicos en el rango de fortaleza $7,2<H_{-}<9,3$, no le confirió al óxido un mejor comportamiento catalítico. Aparentemente los sitios en el intervalo de fortaleza básica $7,2<H<9,3$ no son lo suficientemente fuertes para extraer el protón del alcohol y así generar el ataque nucleofílico al grupo carbonilo del triglicérido, y por tanto no favorecen la transesterificación de triacetina en el mismo grado que los sitios en el intervalo 9,3<H_<15,0. Con los catalizadores de la segunda serie calcinados a $500^{\circ} \mathrm{C}$ se alcanzó conversión total en tan sólo 40 min de reacción. En el caso del $\mathrm{Na} / \mathrm{MgO}-500$ aparecieron sitios de fortaleza básica superior a los del MgO (Fig. 1), que contribuyeron al mejoramiento de la actividad. Sin embargo, su elevada velocidad de reacción podría indicar lixiviación del metal y por tanto se hace necesario verificar su estabilidad. 


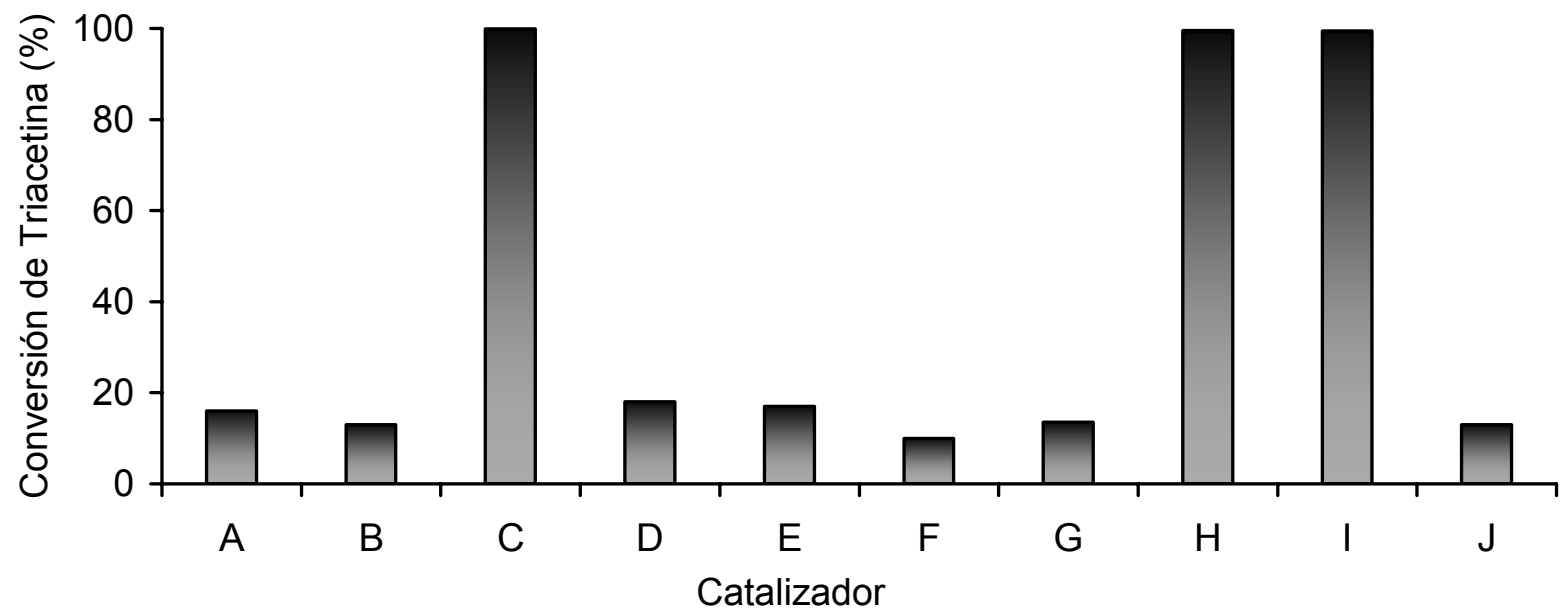

Fig. 2: Conversión final de triacetina con $3 \%$ de catalizador, $60^{\circ} \mathrm{C}$ y 4 h de reacción; $(\mathrm{A}) \mathrm{Na}_{2} \mathrm{CO}_{3}(1) / \gamma$ $\mathrm{Al}_{2} \mathrm{O}_{3},(\mathrm{~B}) \mathrm{KOH}(1) / \gamma-\mathrm{Al}_{2} \mathrm{O}_{3},(\mathrm{C}) \mathrm{CaO},(\mathrm{D}) \mathrm{MgO}_{\mathrm{C}},(\mathrm{E}) \mathrm{MgO},(\mathrm{F}) \mathrm{Cs} / \mathrm{MgO}-900,(\mathrm{G}) \mathrm{Rb} / \mathrm{MgO}-900,(\mathrm{H})$ $\mathrm{Na} / \mathrm{MgO}-500$, (I) Li/MgO-500 y (J) Li/MgO-950.

Con el catalizador Li/MgO-500 no se evidencia correlación alguna entre sus propiedades básicas y texturales, pues a pesar de presentar el área superficial más baja de su serie y un número de sitios básicos intermedio, mostró un excelente desempeño catalítico. Algunos autores han obtenido resultados similares al catalizar otro tipo de reacciones con Li/MgO. Leveles et al. (2002, 2003) trabajaron con Li/MgO, sintetizado por impregnación húmeda, en la conversión oxidativa de alcanos a olefinas; estos autores encontraron que el área del $\mathrm{MgO}\left(30 \mathrm{~m}^{2} / \mathrm{g}\right)$ decrece hasta $6 \mathrm{~m}^{2} / \mathrm{g}$ al incorporar Li. Sin embargo, reportaron que la conversión obtenida con Li/MgO triplicó la alcanzada por el MgO a sus condiciones de operación. De forma semejante Di Cosimo et al. (1996), en un trabajo de síntesis de isoforona por condensación aldólica de acetona con MgO modificado por impregnación húmeda incipiente con $\mathrm{Li}, \mathrm{Na}, \mathrm{K}, \mathrm{Cs}, \mathrm{Ca}, \mathrm{Sr}$ y $\mathrm{Ba}$ como catalizadores, determinaron que la actividad catalítica y la selectividad son mucho más altas para el Li/MgO comparados con los otros miembros de la serie. Los autores plantean que este comportamiento distintivo se puede atribuir al efecto del tamaño iónico, pues la semejanza en los radios iónicos de los cationes $\mathrm{Li}^{+}$y $\mathrm{Mg}^{2+}$ permitiría al $\mathrm{Li}^{+}$reemplazar al $\mathrm{Mg}^{2+}$ en la red del $\mathrm{MgO}$. La sustitución de un ion divalente por uno monovalente en la matriz del $\mathrm{MgO}$ requiere la formación de aniones $\mathrm{O}^{-}$con el fin de mantener la electroneutralidad, resultando en enlaces $\mathrm{Mg}-\mathrm{O}$ tensos y en la formación de especies [ $\mathrm{Li}^{+} \mathrm{O}^{-}$], las cuales actúan como sitios activos del catalizador. En el caso del $\mathrm{Rb}$ y el $\mathrm{Cs}$, sus radios iónicos son mucho mayores al del $\mathrm{Mg}^{2+}$ por tanto, es poco probable que ocurra una modificación estructural de la red del MgO (Di Cosimo et al., 1996). Es posible que estos iones, en su mayoría, se adhieran superficialmente, dificultando la formación de especies activas para la reacción.

A partir de estos resultados es posible asegurar que las propiedades básicas priman sobre las características texturales al determinar el comportamiento catalítico en la transesterificación de triacetina de los sólidos estudiados, y que sólo a partir de un número considerable de sitios básicos de fortaleza $9,3<H_{-}<15,0$ se obtienen altas conversiones de triacetina, pues se observa que la actividad de los catalizadores es fuertemente afectada no sólo por la cantidad de sitios sino también por la fuerza de los sitios básicos. Lo anterior es acorde a lo reportado en la literatura donde demuestran que sólidos con fortaleza básica dentro del rango $9,3<H_{-}<15,0$ y en rangos superiores, actúan de forma eficiente en la transesterificación de triglicéridos (Xie et al., 2006). Kawashima et al. (2009) estudiaron la transesterificación de aceite de colza con $\mathrm{CaO}$ activado con metanol, de fortaleza básica 10,1<H_<11,1, alcanzando una producción de $90 \%$ de metil éster en $3 \mathrm{~h}$ a $60^{\circ} \mathrm{C}$.

Los catalizadores de la segunda serie que presentaron baja actividad catalítica se sometieron a activación en flujo de $\mathrm{N}_{2}$. Los resultados se presentan en la Fig. 3. Se observa que al activar los catalizadores la velocidad de reacción y la conversión de triacetina aumentó. Se presenta aproximadamente el doble de actividad comparada con la de los mismos sin activar. El comportamiento mostrado por los catalizadores después de la activación se puede atribuir al efecto de la eliminación de los contaminantes ácidos que inhiben los sitios de reacción, como ha sido 
sugerido en la literatura (Dossin et al., 2006). En la activación, las altas temperaturas y el contacto prolongado de la superficie con nitrógeno promueven la desorción de ácidos débiles, como el $\mathrm{CO}_{2}$, adsorbidos sobre los sitios activos (Arzamendi et al., 2008).

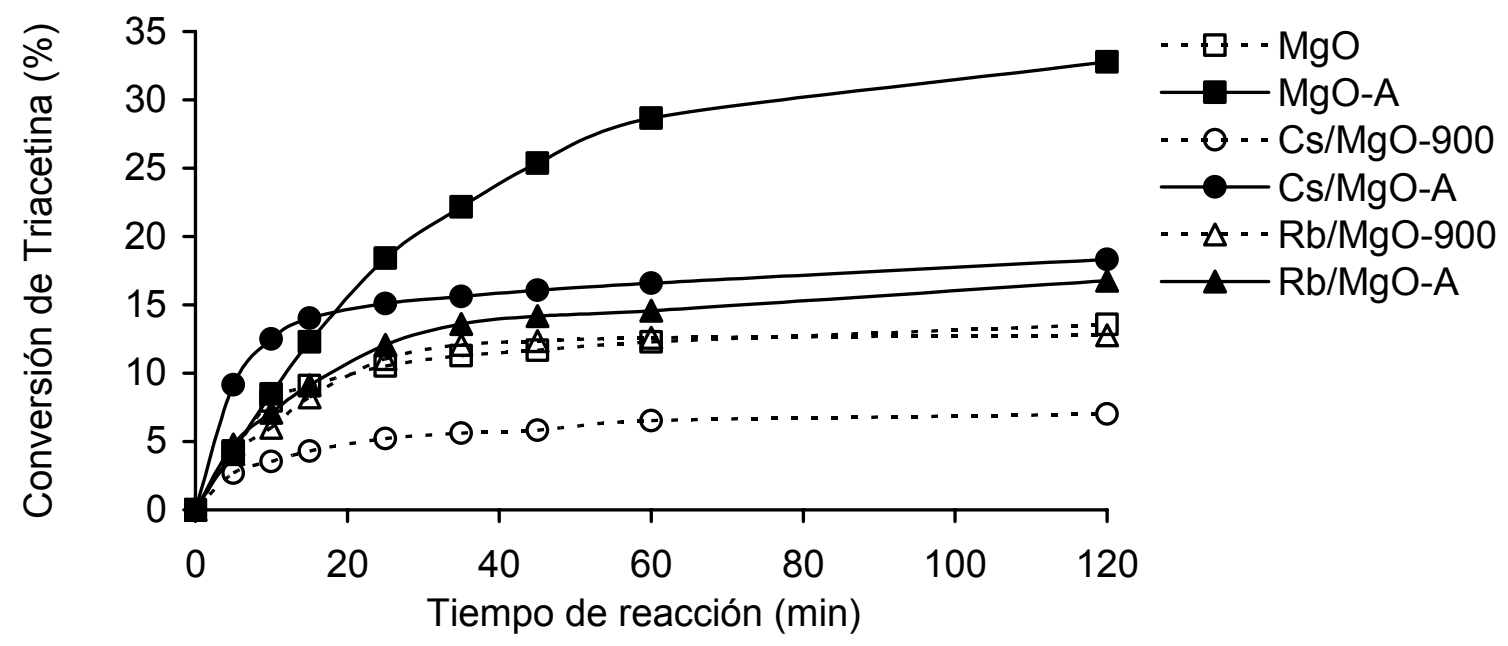

Fig. 3: Influencia de la activación con $\mathrm{N}_{2}$ sobre la actividad en la transesterificación de triacetina

Se analizó la estabilidad de los catalizadores mediante su reuso en la reacción. Con el Cs/MgO-A la conversión disminuyó de $18 \%$ en la primera reacción a $8 \%$ en el reuso y con el $\mathrm{Rb} / \mathrm{MgO}-\mathrm{A}$ de $15 \%$ a $4 \%$. En el caso del Li/MgO-950 la conversión con catalizador fresco y usado es alrededor del $13 \%$ en ambos casos; se puede afirmar que el tratamiento a alta temperatura no condujo a una actividad aceptable pero garantizó la estabilidad. En la Fig. 4 se muestran los resultados obtenidos con los catalizadores que presentaron mejor desempeño catalítico: $\mathrm{CaO}, \mathrm{Na} / \mathrm{MgO}-500$ y Li/MgO-500 frescos y usados, estos últimos identificados con la letra $\mathrm{R}$ al final de su sigla.

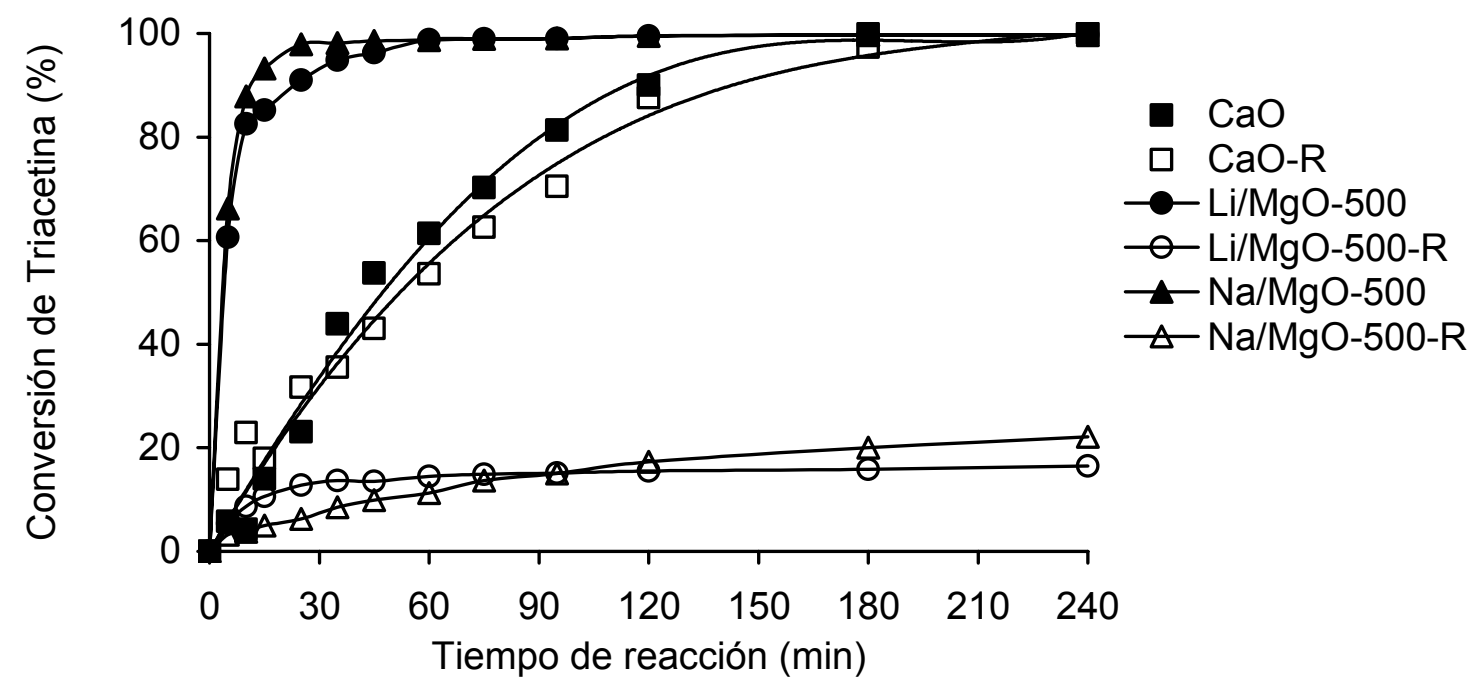

Fig. 4: Actividad de los catalizadores frescos y usados

En las reacciones de reuso los catalizadores resultantes después de la impregnación de metales alcalinos no alcanzan una conversión igual o cercana a la lograda en su primer uso, indicando que no retienen el metal. Se comprueba que el alto desempeño catalítico presentado por los catalizadores frescos impregnados con metales es atribuido a la lixiviación del metal, que conlleva a la presencia de iones metálicos disueltos y por tanto, la reacción se lleva a cabo en fase homogénea. Se destaca en la Fig. 4 que el $\mathrm{CaO}$ reutilizado presenta la misma actividad que el $\mathrm{CaO}$ fresco, alcanzando en ambos casos conversión completa en aproximadamente $3 \mathrm{~h}$ de reacción. 
Para analizar más a fondo la estabilidad del $\mathrm{CaO}$ se determinó su grado de disolución en el medio de reacción por A.A: se disolvió sólo el $1,7 \%$ del $\mathrm{CaO}$ empleado al inicio de la reacción. Para verificar si el Ca detectado en solución participó en la reacción, el catalizador fue removido por centrifugación y filtración al finalizar la reacción. Se agregó triacetina a la solución filtrada y se comprobó que no hubo conversión durante $1 \mathrm{~h}$ a las condiciones de reacción, confirmando que la catálisis sólo estuvo asociada a la fase sólida del material. Además, el $\mathrm{CaO}$ se sometió a 5 ciclos de reacción y se observó que no perdió su actividad, alcanzando conversiones del mismo orden (>90\%) en todos los ciclos. Por su alta actividad catalítica, estabilidad y fácil separación de los productos de reacción, el $\mathrm{CaO}$ se muestra en este estudio como el mejor catalizador entre los sólidos ensayados.

En la Fig. 5 se presentan los resultados de selectividad para el CaO en función de la conversión de triacetina a las condiciones de reacción. Los resultados son consistentes con el esquema de reacción que ha sido propuesto por López et al. (2005), donde los diglicéridos son los primeros productos en formarse y por tanto la selectividad hacia la diacetina a bajas conversiones es cercana a 100\%. Esta selectividad decrece rápidamente mientras se produce glicerina y el éster etílico. La monoacetina no alcanza altos valores de selectividad y presenta una tendencia a desaparecer con el tiempo de reacción, mientras que la glicerina, que aparece al alcanzar aproximadamente $30 \%$ de conversión, tiende a alcanzar $100 \%$ de selectividad a medida que avanza la reacción.

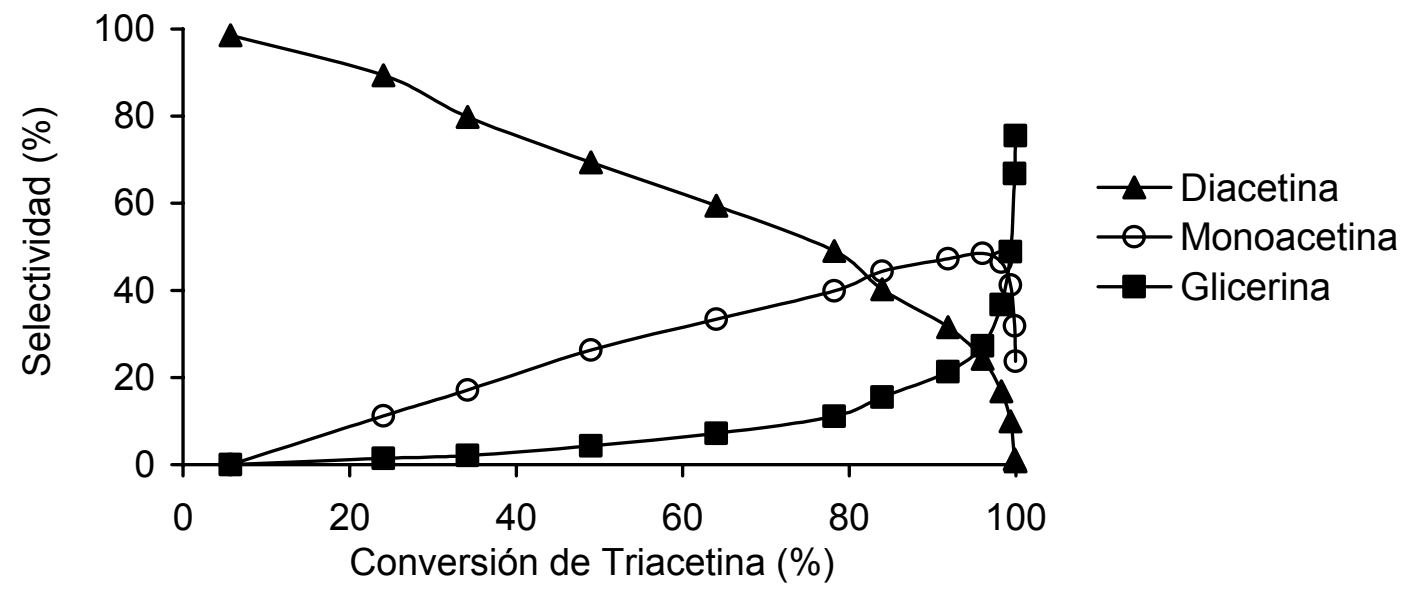

Fig. 5: Selectividad hacia diacetina, monoacetina y glicerina en función de la conversión de triacetina a $60^{\circ} \mathrm{C}$ y $3 \%$ de $\mathrm{CaO}$.

\section{CONCLUSIONES}

De este estudio pueden extraerse las siguientes conclusiones: (1) las propiedades básicas de los catalizadores estudiados, a diferencia de las características texturales, influyen de forma significativa en la actividad catalítica en la reacción de transesterificación de triacetina; (2) la activación con $\mathrm{N}_{2}$ a $500^{\circ} \mathrm{C}$ para los catalizadores sintetizados a partir de $\mathrm{MgO}$, incrementa la conversión de triacetina y por tanto, la producción del éster etílico; (3) ni la impregnación de $\gamma-\mathrm{Al}_{2} \mathrm{O}_{3}$ con $\mathrm{Na}$ y $\mathrm{K}$ ni la modificación del $\mathrm{MgO}$ con $\mathrm{Na}, \mathrm{Li}, \mathrm{Rb}$ y $\mathrm{Cs}$ conducen a sólidos básicos estables. La actividad mostrada por estos catalizadores en reacciones de transesterificación se debe a la presencia de iones metálicos disueltos, por lo tanto la reacción en estos casos se lleva a cabo en fase homogénea, y finalmente (4) los resultados experimentales demuestran que el $\mathrm{CaO}$, con una fortaleza básica de $7,2<H_{-}<9,3$ y $9,3<H_{-}<15,0$ y sin ningún tratamiento adicional, es un buen catalizador para la transesterificación de triglicéridos ya que presenta unas excelentes actividad y estabilidad en esta reacción.

\section{AGRADECIMIENTOS}

Los autores agradecen a la Vicerrectoría de Investigación y Extensión de la UIS por el soporte financiero a través del proyecto código 5423, y a COLCIENCIAS por la beca otorgada a M. Becerra mediante el programa “Jóvenes Investigadores" durante sus estudios de Maestría en Ing. Química. 


\section{REFERENCIAS}

Arzamendi, G. y otros cinco autores, Synthesis of biodiesel with heterogeneous NaOH/alumina catalysts: Comparison with homogeneous NaOH, Chem. Eng. J., 134, 123-130 (2007).

Arzamendi, G. y otros cuatro autores, Alkaline and alkaline-earth metals compounds as catalysts for methanolysis of sunflower oil, Catal. Today, 133, 305-313 (2008).

Bailly, M.-L. y otros cinco autores, A spectroscopy and catalysis study of the nature of active sites of $\mathrm{MgO}$ catalysts: Thermodynamic Brønsted basicity versus reactivity of basic sites, J. Catal., 235, 413422 (2005).

Di Cosimo, J.I., V.K. Díez y C.R. Apesteguía, Base catalysis for the synthesis of $\alpha, \beta$-unsaturated ketones from the vapor-phase aldol condensation of acetone, Appl. Catal. A, 137(1), 149-166 (1996).

Díez, V.K., C.R. Apesteguía y J.I. Di Cosimo, Acid-base properties and active site requirements for elimination reactions on alkali-promoted MgO catalysts, Catal. Today, 63, 53-62 (2000).

Dossin, T.F, M.-F. Reyniers y G.B. Marin, Kinetics of heterogeneously MgO-catalyzed transesterification, Appl. Catal. B, 61, 35-45 (2006).

Ebiura, T. y otros cuatro autores, Selective transesterification of triolein with methanol to methyl oleate and glycerol using alumina loaded with alkali metal salt as a solid-base catalyst, Appl. Catal. A, 283, 111-116 (2005).

Hattori, H., Solid base catalysts: Generation, characterization, and catalityc behavior of basic sites, J. Jpn. Petrol. Inst., 47, 67-81 (2004).

Hee Lee, M. y D. Gon Park, Preparation of MgO with high surface area, and modification of its pore characteristics, Bull. Korean Chem. Soc., 24(10), 1437-1443 (2003).

Kawashima, A., K. Matsubara y K. Honda, Acceleration of catalytic activity of calcium oxide for biodiesel production, Bioresour. Technol, 100, 696-700 (2009).

Kim, H-J. y otros seis autores, Transesterification of vegetable oil to biodiesel using heterogeneous base catalyst, Catal. Today, 93-95, 315-320 (2004).

Kouzu, M. y otros cinco autores, Calcium oxide as a solid base catalyst for transesterification of soybean oil and its application to biodiesel production, Fuel, 87, 2798-2806 (2008).

Leveles, L. y otros cuatro autores, Oxidative conversion of light alkanes to olefins over alkali promoted oxide catalysts, Appl. Catal. A, 227, 287-297 (2002).

Leveles, L. y otros tres autores, Oxidative conversion of propane over lithium-promoted magnesia catalyst II. Active site characterization and hydrocarbon activation, J. Catal., 218, 307-314 (2003).

López, D.E. y otros tres autores, Transesterification of triacetin with metanol on solid acid and base catalysts, Appl. Catal. A, 295(2), 97-105 (2005).

Trionfetti, C. y otros tres autores, Formation of high surface area Li/MgO-Efficient catalyst for the oxidative dehydrogenation/cracking of propane, Appl. Catal. A, 310, 105-113 (2006).

Xi, Y. y R.J. Davis, Influence of water on the activity and stability of activated Mg-Al hydrotalcites for the transesterification of tributyrin with methanol, J. Catal., 254, 190-197 (2008).

Xie, W. y H. Li, Alumina-supported potassium iodide as a heterogeneous catalyst for biodiesel production from soybean oil, J. Mol. Catal. A: Chem., 255, 1-9 (2006). 